\title{
Spectroscopic analysis of high electric field enhanced ionization in laser filaments in air for corona guiding
}

\author{
Yingxia Wei ${ }^{1}$, Yaoxiang $\mathrm{Liu}^{1}$, Tie-Jun Wang ${ }^{1}$, Na $\mathrm{Chen}^{1}$, Jingjing $\mathrm{Ju}^{1}$, Yonghong $\mathrm{Liu}^{1}$, Haiyi Sun ${ }^{1}$, \\ Cheng Wang ${ }^{1}$, Jiansheng Liu ${ }^{1}$, Haihe $\mathrm{Lu}^{1}$, See Leang Chin ${ }^{2}$, and Ruxin $\mathrm{Li}^{1}$ \\ ${ }^{1}$ State Key Laboratory of High Field Laser Physics, Shanghai Institute of Optics and Fine Mechanics, \\ Chinese Academy of Sciences, China \\ ${ }^{2}$ Centre d'Optique, Photonique et Laser (COPL) and Département de physique, de génie physique et d'optique, \\ Université Laval, Québec, Québec G1V OA6, Canada \\ (Received 20 December 2015; revised 27 January 2016; accepted 4 February 2016)
}

\begin{abstract}
We report on a systematic experimental study on the fluorescence spectra produced from a femtosecond laser filament in air under a high electric field. The electric field alone was strong enough to create corona discharge (CD). Fluorescence spectra from neutral and ionic air molecules were measured and compared with pure high-voltage CD and pure laser filamentation (FIL). Among them, high electric field assisted laser FIL produced nitrogen fluorescence more efficiently than either pure CD or pure FIL processes. The nonlinear enhancement of fluorescence from the interaction of the laser filament and corona discharging electric field resulted in a more efficient ionization along the laser filament zone, which was confirmed by the spectroscopic measurement of both ionization-induced fluorescence and plasma-scattered $800 \mathrm{~nm}$ laser pulses. This is believed to be the key precursor process for filament-guided discharge.
\end{abstract}

Keywords: femtosecond laser filamentation; filament induced fluorescence spectroscopy; laser guided discharges

\section{Introduction}

Filamentation (FIL) induced by ultrashort intense laser pulses in air has attracted much scientific interest since it was first predicted theoretically in $1990^{[1]}$ and first observed experimentally in $1995^{[2]}$. The main physical mechanism of femtosecond laser FIL is well known to be a dynamic equilibrium between self-focusing by the optical Kerr effect and defocusing by a self-generated plasma produced by multiphoton/tunnel ionization ${ }^{[3-5]}$. During femtosecond laser FIL in air, many nonlinear effects occur simultaneously, such as intensity clamping, molecular alignment, harmonic generation, self-phase modulation and so on ${ }^{[3-5]}$. These complex and interesting nonlinear phenomena accompanying femtosecond laser FIL lead to promising applications in different fields, such as remote sensing ${ }^{[6,7]}$, few-cycle pulse compression ${ }^{[8]}$, atmospheric condensation and precipitation ${ }^{[9-11]}$, secondary light source generation ranging from air lasing to terahertz emission ${ }^{[12-18]}$, and triggering and guiding high-voltage discharge with possible applications in lightning control ${ }^{[19-24]}$.

Correspondence to: T.-J. Wang, No. 390 Qinghe Road, Jiading District, Shanghai, 201899, China. Email: tiejunwang@ siom.ac.cn
Triggering and guiding lightning using lasers was proposed by scientists in $1974^{[25]}$. Four years later in 1978, Greig et al. ${ }^{[26]}$ utilized high-energy $\mathrm{CO}_{2}$ and YAG lasers to investigate laser-guided discharges. Much effort was devoted to understanding the fundamental mechanism of lasertriggered lightning in the $1980 \mathrm{~s}^{[27-29]}$. Despite several successful examples for real-scale observation of laser-triggered lightning reported by a Japanese group in the late $1990 \mathrm{~s}^{[30]}$, this method was progressively abandoned because of the discontinuous profile of the plasma generated by such long pulses through avalanche breakdown. With the invention of chirped pulse amplification (CPA) technology in $1985^{[31]}$, the output power of femtosecond lasers has been rapidly improved. A plasma channel over a long distance induced by femtosecond laser, namely a laser filament, made a great contribution in this field. The plasma density of an air filament is higher than $10^{15} \mathrm{~cm}^{-3}$, leading to a conducting channel (low resistivity) formed in air. The possibility of using a laser filament to couple the external electromagnetic fields and initiate the high-voltage discharge ${ }^{[32]}$ has been successfully demonstrated ${ }^{[33]}$. Although there are many experimental contributions on filament-guided discharges ${ }^{[19-23,32-36]}$, investigations on how a laser filament guides the external high-voltage corona discharge (CD) had not been fully 


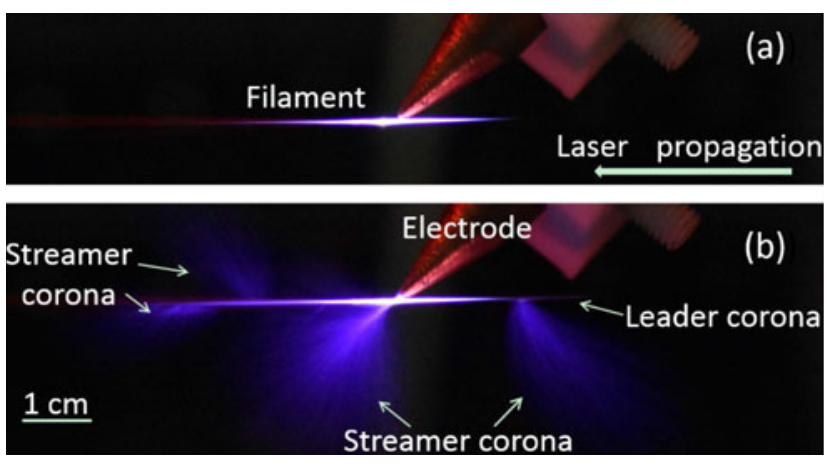

Figure 1. Real-color image of (a) a typical filament and (b) filament-guided CDs taken by a digital camera. The CD voltage and filamenting pulse energy were $50 \mathrm{kV}$ and $5.4 \mathrm{~mJ}$, respectively.

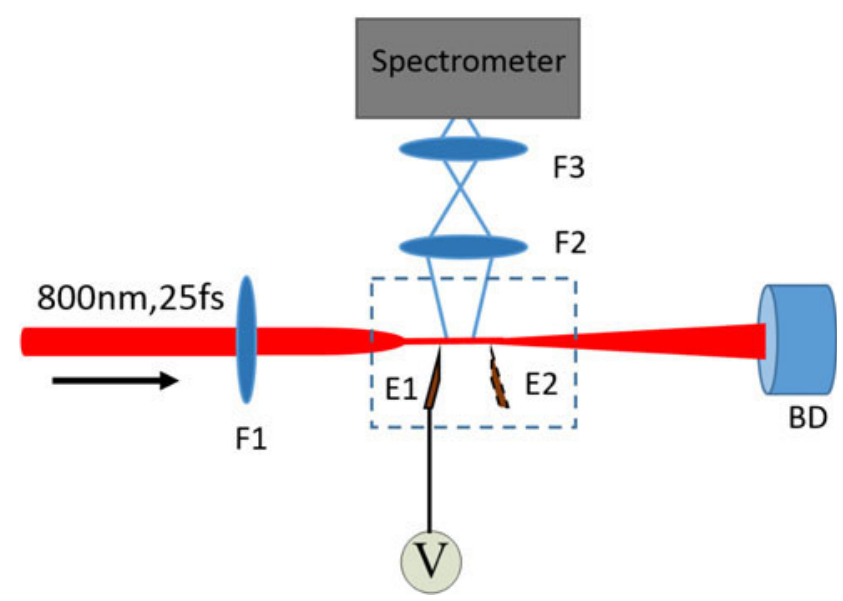

Figure 2. Side schematic of the experimental setup for fluorescence detection in air. F1-F3 are focusing UV grade fused silica lenses with focal lengths of 30, 15 and $10 \mathrm{~cm}$, respectively. E1 and E2 are electrodes. E1 was connected to a high-voltage power supply and E2 was floated. BD is a beam dump.

explored yet until recently, when we reported on a direct observation of laser-filament-guided $\mathrm{CDs}^{[37]}$. When a laser filament was generated at $\sim 1 \mathrm{~mm}$ from the tip of a copper electrode, as shown in Figure 1(a), a high DC voltage of $50 \mathrm{kV}$ could be guided along the laser filament, forming blue CDs on both ends of the filament. Both leader and streamer types of corona guided by laser filaments have been observed, as shown in Figure 1(b).

Following our first observation of laser-guided $\mathrm{CD}^{[37]}$, in this work we provide a systematic investigation of the interaction between a laser filament and high-voltage CD by looking at the spectroscopic behavior of the fluorescence from the filament. High-voltage electric field enhanced ionization along the laser filament was observed through ionization-induced fluorescence measurements and was confirmed by the measurement of plasma-scattered $800 \mathrm{~nm}$ laser pulses. This is believed to be the key process for corona guiding high-voltage discharge through laser FIL.

\section{Experimental method and setup}

The experimental setup is schematically presented in Figure 2. A pair of copper electrodes with $1 \mathrm{~mm}$ diameter tips was used in the experiment. The separation distance between the two tips was variable. One electrode (E1) was connected to a DC high-voltage power supply with output up to $100 \mathrm{kV} / 1000 \mathrm{~W}$. The other electrode (E2) was floated. A $1 \mathrm{kHz} / 25 \mathrm{fs} / 800 \mathrm{~nm}$ laser pulse from a Ti:sapphire (CPA) system (beam diameter $\sim 15 \mathrm{~mm}$ ) was focused by a $30 \mathrm{~cm}$ focal length plano-convex lens, producing a filament centered approximately $1-2 \mathrm{~mm}$ above the tips. The femtosecond laser pulse has a maximum energy of $8.0 \mathrm{~mJ}$. The fluorescence signal produced by the femtosecond laser filament with and without high-voltage $\mathrm{CD}$ was collected by a pair of lenses and sent to a spectrometer coupled to a CCD camera. Note that the second electrode (E2) was floated in order to maintain the $\mathrm{CD}$ and to investigate the spectroscopic behavior of the interaction between the laser filament and high-voltage $\mathrm{CD}$ more easily. An image of the tips of the two electrodes is shown in Figure 3(a).

\section{Experimental results and analysis}

\subsection{Laser-guided leader type of corona}

In the fluorescence image measurements of Figure 3, the filamenting pulse energy and CD voltage were fixed at $4.8 \mathrm{~mJ}$ and $50 \mathrm{kV}$, respectively. The distance between the electrode tips was set at $\sim 15 \mathrm{~mm}$. The fluorescence length of the filament was $\sim 10 \mathrm{~mm}$, which is shorter than the separation distance between the two tips of $\sim 15 \mathrm{~mm}$. In order to make a comparison, the images of CD, FIL and high-voltage electric field biased filamentation (eFIL) were taken under the same camera condition. Typical fluorescence images recorded by a high-speed camera are shown in Figures 3(b)3(d). The fluorescence from CD was too weak to be detected (Figure 3(b)). The fluorescence images of FIL and eFIL are shown in Figures 3(c) and 3(d), respectively. The strong signals on the two electrodes (white areas in Figure 3(c) and 3(d) on the electrodes) were due to the strong scattered laser light. The plasma filament itself could not bridge the two electrodes (Figure 3(c)). The plasma channel induced by the laser filament under the high-voltage electric field could easily connect the two electrodes (Figure 3(d)). This is also evidence of a plasma-filament-guided leader type of corona.

\subsection{Fluorescence}

In the following measurements, the distance between the tips of the electrodes was set at $\sim 7.5 \mathrm{~mm}$ so as to sample the strongest part of the filament. Three types of typical fluorescence spectra, namely from CD, FIL and eFIL, with 


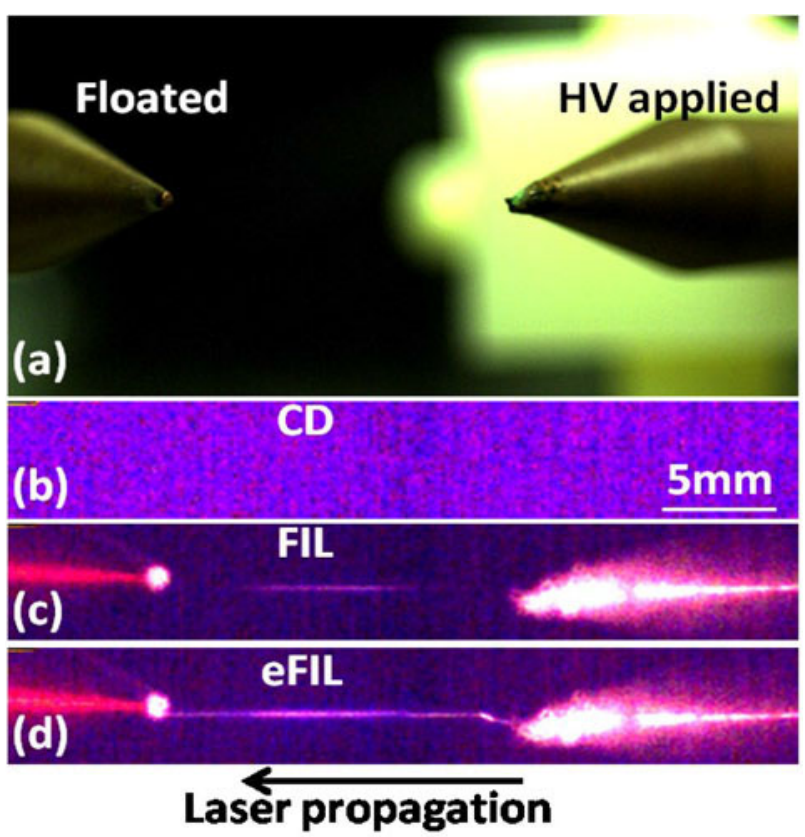

Figure 3. Laser-guided leader type of $\mathrm{CD}$. (a) Image of the tips of the two electrodes and typical fluorescence images of (b) CD, (c) FIL and (d) eFIL. The separation distance of the two tips was $\sim 15 \mathrm{~mm}$. The filamenting pulse energy was $4.8 \mathrm{~mJ}$ and the $\mathrm{CD}$ voltage was $50 \mathrm{kV}$. The fluorescence length of the filament was $\sim 10 \mathrm{~mm}$. It was situated at approximately $1-2 \mathrm{~mm}$ above the tips of the electrodes.

spectra ranging from the UV to VIS-NIR were measured. The results are shown in Figure 4. In these measurements, the voltage for $\mathrm{CD}$ and the laser pulse energy for FIL in the three cases were fixed at $50 \mathrm{kV}$ and $7.0 \mathrm{~mJ}$, respectively. Note that there was CD spreading out from the floated electrode even without the laser filament. That emission was not collected in this measurement since our attention here was on the filament zone under a high voltage, which would provide information on the ionization process and, hence, the conducting property of the laser filament.

Figure 4(a) (Ref. [37]) shows typical fluorescence spectra in the $290-440 \mathrm{~nm}$ region covering the signals from the first negative band system of $\mathrm{N}_{2}^{+}\left(B^{2} \Sigma_{u}^{+}-X^{2} \Sigma_{g}^{+}\right.$transition) and the second positive band system of $\mathrm{N}_{2}\left(C^{3} \Pi_{u}-B^{3} \Pi_{g}\right.$ transition $)^{[38,39]}$. It shows that the structures of the spectrum from molecular $\mathrm{N}_{2}$ are similar in the three cases, but different at 391 and $428 \mathrm{~nm}$, which are ionic lines from excited $\mathrm{N}_{2}^{+}$through the transitions of $B^{2} \Sigma_{u}^{+}(0)-X^{2} \Sigma_{g}^{+}(0)$ and $B^{2} \Sigma_{u}^{+}(0)-X^{2} \Sigma_{g}^{+}(1)$. In the cases of FIL and eFIL, the spectral intensities at these two ionic lines are very strong. However, they are too weak to be distinguished from the background in the case of $\mathrm{CD}$. This difference may be due to the different generation processes.

In the case of $\mathrm{CD}$, the fluorescence is mainly generated by avalanche ionization through collision processes, in which the probability to populate $\mathrm{N}_{2}^{+}$to an excited state is very low as compared to exciting neutral $\mathrm{N}_{2}$. In the case
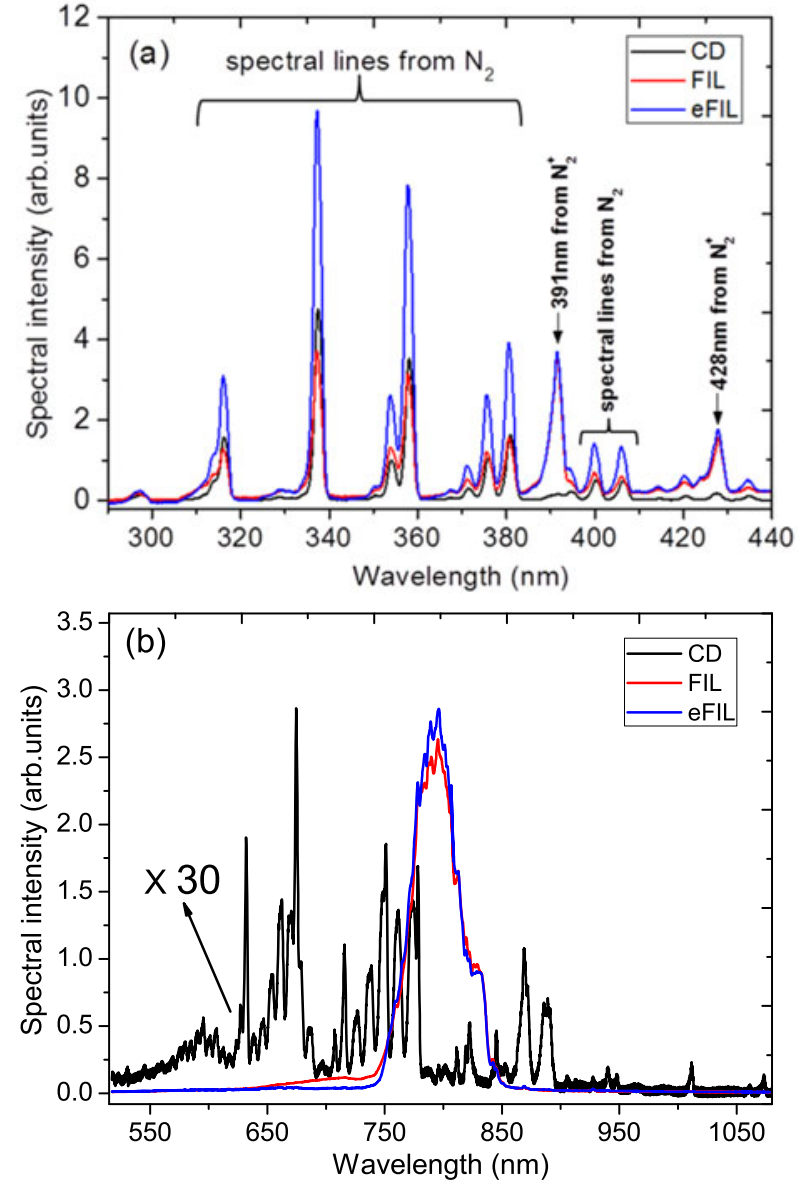

Figure 4. Typical spectrum in the range (a) 290-440 nm (Ref. [37]) and (b) $500-1090 \mathrm{~nm}$ emitted by CD, FIL and eFIL, respectively. The intensity of CD fluorescence is magnified by 30 times in (b).

of FIL, $\mathrm{N}_{2}^{+}$are generated by multiphoton/tunneling ionization. The laser intensity inside the laser filament is high enough to ionize $\mathrm{N}_{2}$ and excite $\mathrm{N}_{2}^{+}$into $B^{2} \Sigma_{u}^{+}$, leading to ionic fluorescence. Then, through electron collision and dissociative recombination processes, e.g. $\mathrm{N}_{2}^{+}+\mathrm{N}_{2} \rightarrow$ $\mathrm{N}_{4}^{+} ; \mathrm{N}_{4}^{+}+\mathrm{e} \rightarrow \mathrm{N}_{2}^{*}+\mathrm{N}_{2} ; \mathrm{N}_{2}^{*} \rightarrow \mathrm{N}_{2}+h v$ (337, 357, $380 \mathrm{~nm}$ etc. $)^{[38]}$, excited $\mathrm{N}_{2}$ can be populated, leading to $\mathrm{N}_{2}$ fluorescence emission. Hence, any increase in the fluorescence from $\mathrm{N}_{2}$, such as the $337 \mathrm{~nm}$ line, would indicate an increase in the number of $\mathrm{N}_{2}^{+}$; i.e. an increase of ionization. Indeed, Figure 4(a) shows that the fluorescence from neutral $\mathrm{N}_{2}$ is highest in the case of eFIL. That is to say, more ionization occurred in the filament zone in eFIL; hence, the plasma density is higher in eFIL.

In addition, the spectral intensities at 391 and $428 \mathrm{~nm}$ from eFIL are almost equal to that from FIL. This indicates that they might come mostly from the same contribution as FIL. That is to say, multiphoton/tunneling ionization in a filament contributes more to the ionic fluorescence as compared to the electron impact ionization in the case of $\mathrm{CD}$. 

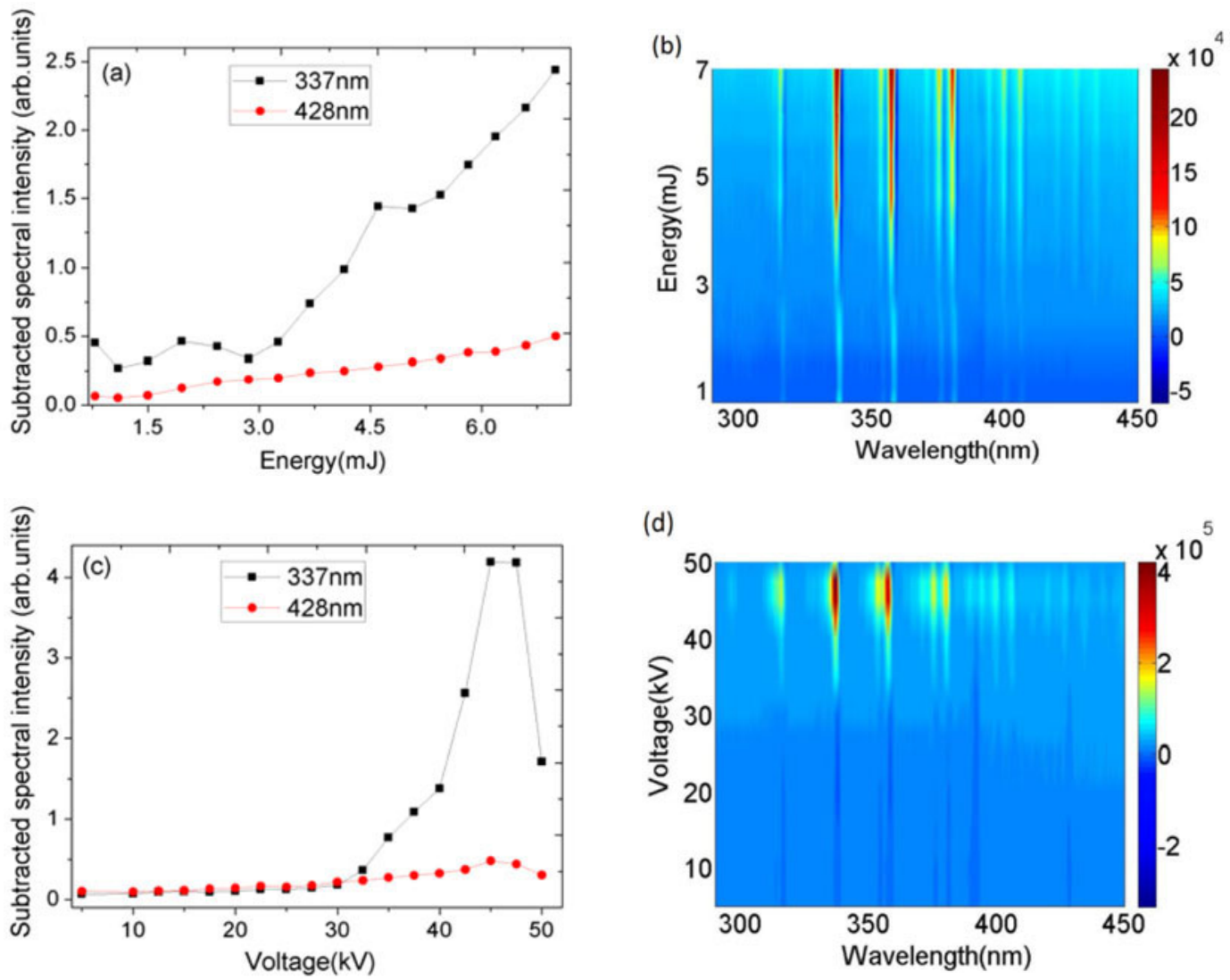

Figure 5. (a) Resultant spectral intensity as a function of the pulse energy at 337 and $428 \mathrm{~nm}$. The CD voltage was fixed at $50 \mathrm{kV}$. (b) Pseudocolor plot of pump energy versus wavelength corresponding to (a). (c) Resultant spectral intensity as a function of CD voltage when the pulse energy was fixed at $4.61 \mathrm{~mJ}$. (d) Pseudocolor plot of voltage versus wavelength corresponding to (c).

In Figure 4(b), in the wavelength range 500-1090 nm, the weak intensity of $\mathrm{CD}$ fluorescence is magnified by a factor of 30. Because of the high scattered intensity around $800 \mathrm{~nm}$ in the cases of FIL and eFIL, it is difficult to recognize this pure CD fluorescence structure without expanding it. The scattered $800 \mathrm{~nm}$ signal by eFIL is stronger than that by FIL. This again indicates that the plasma density in eFIL is higher than that in FIL.

We now look at the dependence of fluorescence of eFIL on the laser pulse energy and the voltage. At a fixed voltage of $\mathrm{CD}$, the fluorescence signal of eFIL as a function of filamenting pulse energy was measured. Then the $\mathrm{CD}$ and FIL fluorescence signals were subtracted from the eFIL signal.

Figure 5(a) depicts the resultant signal intensity at 337 and $428 \mathrm{~nm}$ as a function of the pulse energy when the $\mathrm{CD}$ voltage was fixed at $50 \mathrm{kV}$. Figure $5(\mathrm{~b})$ shows the pseudocolor plot $^{[37]}$ in the spectral region corresponding to Figure 5(a). A clear positive resultant signal was obtained, which indicates that the resultant (extra) fluorescence came from the interaction. In particular, this fluorescence becomes much stronger when using a high filamenting pulse energy, probably because the filament length becomes longer.
Among them, the increase of molecular $\mathrm{N}_{2}$ fluorescence is much more than that from ionic $\mathrm{N}_{2}^{+}$. It means that, under high voltage, the plasma density in the filament zone increased as the laser pulse energy increased.

In another measurement, the resultant eFIL fluorescence signal as a function of $\mathrm{CD}$ voltage was recorded when the filamenting pulse energy was fixed at $4.61 \mathrm{~mJ}$. The length of the filament at this energy $(\sim 1 \mathrm{~cm})$ was enough to connect two electrodes spaced by $7.5 \mathrm{~mm}$. The resultant signal intensity and its pseudocolor plot are shown in Figures 5(c) and 5(d), respectively. An enhancement of fluorescence signal similar to that shown in Figures 5(a) and 5(b) was observed. In particular, the fluorescence from neutral $\mathrm{N}_{2}$ $(337 \mathrm{~nm})$ becomes much stronger at higher voltages. This again indicates that the plasma density in the eFIL zone became higher at higher $C D$ voltage. Note that, when the voltage applied on the first electrode E1 was too high, the filament-guided high-voltage electric field on the second electrode could lead to $\mathrm{CD}$ at the tail of the second electrode (floated). Under this circumstance, the fluorescence from the tail of the second electrode could not be collected because it was outside the measurement range. This could be responsible for the signal saturation, and even decrease, when the voltage was higher than $\sim 45 \mathrm{kV}$ (Figure 5(c)). 

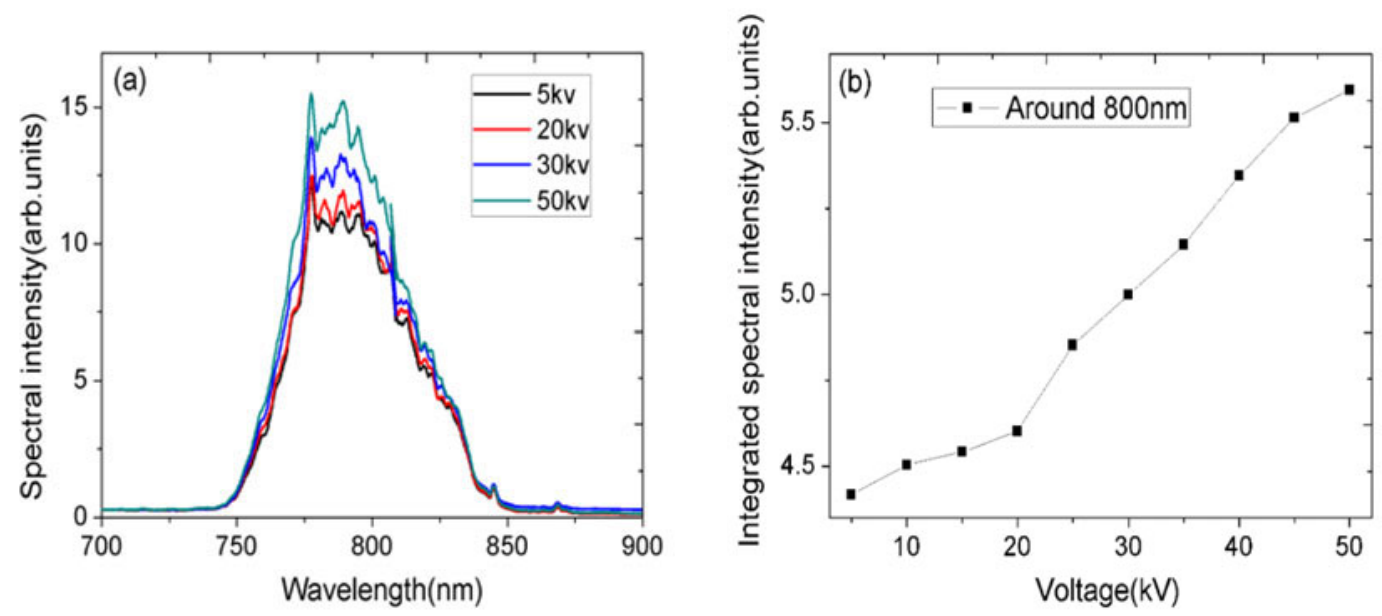

Figure 6. (a) Spectrum in the range 700-900 nm at different CD voltages: 5, 20, 30 and $50 \mathrm{kV}$, respectively. (b) Integrated spectral intensity as a function of CD voltage. The integrated spectral range is from 745 to $855 \mathrm{~nm}$. The filamenting pulse energy was fixed at $4.61 \mathrm{~mJ}$.

The fluorescence enhancement in the nonlinear interaction of FIL and CD is due to the enhanced ionization along the laser filament. When the $\mathrm{CD}$ voltage is fixed, a higherdensity plasma is generated by increasing the filamenting pulse energy. When the high-voltage electric field through the electrode was applied to the filament, more free electrons inside the hot filament zone would be accelerated towards the electrode, enhancing the ionization along the laser filament. This would enhance the neutral $\mathrm{N}_{2}$ fluorescence through collisional processes ${ }^{[38]}$. In the case of fixed filamenting pulse energy, a higher $\mathrm{CD}$ voltage will provide a stronger electric field. The higher the $\mathrm{CD}$ voltage is, the faster the free electrons move. As a consequence, stronger ionization through collisions can be generated along the laser filament. Hence, a stronger fluorescence can be detected.

One more experiment was carried out by measuring the side-scattered spectrum around $800 \mathrm{~nm}$ (pump laser central wavelength) at different CD supply voltages from 5 to $50 \mathrm{kV}$ when the pump pulse energy was fixed at $4.61 \mathrm{~mJ}$. Because of the scattering effect of ionization-induced plasmas, the emission from inside the filament zone should be scattered out. As shown in Figure 6(a), the scattered signal at $800 \mathrm{~nm}$ increases as the $\mathrm{CD}$ voltage increases. It is proportional to the CD voltage (Figure 6(b)). Again, it experimentally confirms that more ionization was generated along the laser filament when increasing the voltage or the energy of the femtosecond laser pulse.

\section{Discussion and conclusion}

The results reported in this paper provide a clear physical picture of the observed laser-filament-guided $\mathrm{CD}^{[37]}$. They show that there was more ionization of nitrogen molecules inside the filament zone when a high voltage was applied to the filament. Since the ionization potential of a nitrogen molecule $(15.576 \mathrm{eV})$ is higher than that of an oxygen molecule $(12.06 \mathrm{eV})$, it is expected that many more oxygen molecules were ionized. This would enhance the conductivity of the filament zone. When the high (positive) voltage electric field through the electrode was applied to the filament, the free electrons inside the hot filament zone would be accelerated towards the electrode, enhancing the ionization along their path. This would enhance the fluorescence. At the same time, because electrons are pushed and/or attracted to the electrode at a high positive voltage, the filament would become positively charged. The two sharp ends of the filament would accumulate more positive charges, giving rise to a high field. The strong electric fields at the two sharp extremities of the filament would then induce local CDs linking the filament with the two electrodes, as shown in Figure 3(d). Such CDs of the filament zone would be the precursor to induce a full discharge between two electrodes set at a high voltage difference $^{[19-23,32-36]}$.

\section{Acknowledgments}

This work was supported in part by National Natural Science Foundation of China (Nos 61221064, 11127901 and 11404354), the National 973 Project of China (No. 2011CB808103), the Chinese Academy of Sciences and the State Key Laboratory of High Field Laser Physics, the 100 Talents Program of Chinese Academy of Sciences, and the Shanghai Pujiang Program.

\section{References}

1. Y. Silberberg, Opt. Lett. 15, 1282 (1990).

2. A. Braun, G. Korn, X. Liu, D. Du, J. Squier, and G. Mourou, Opt. Lett. 20, 73 (1995). 
3. S. L. Chin, Femtosecond Laser Filamentation (Springer, New York, 2010).

4. S. L. Chin, T.-J. Wang, C. Marceau, J. Wu, J. S. Liu, O. Kosareva, N. Panov, Y. P. Chen, J.-F. Daigle, S. Yuan, A. Azarm, W. W. Liu, T. Seideman, H. P. Zeng, M. Richardson, R. Li, and Z. Z. Xu, Laser Phys. 22, 1 (2012).

5. A. Couairon and A. Mysyrowicz, Phys. Rep. 441, 47 (2007).

6. S. L. Chin, H. L. Xu, Q. Luo, F. Théberge, W. Liu, J.-F. Daigle, Y. Kamali, P. T. Simard, J. Bernhardt, S. A. Hosseini, M. Sharifi, G. Méjean, A. Azarm, C. Marceau, O. Kosareva, V. P. Kandidov, N. Aközbek, A. Becker, G. Roy, P. Mathieu, J. R. Simard, M. Châteauneuf, and J. Dubois, Appl. Phys. B 95, 1 (2009).

7. H. Xu, Y. Cheng, S.-L. Chin, and HongBo Sun, Laser Photon. Rev. 9, 275 (2015).

8. C. P. Hauri, W. Kornelis, F. W. Helbing, A. Heinrich, A. Couairon, A. Mysyrowicz, J. Biegert, and U. Keller, Appl. Phys. B 79, 673 (2004).

9. J. Ju, J. Liu, C. Wang, H. Sun, W. Wang, X. Ge, C. Li, S. L. Chin, R. Li, and Z. Xu, Opt. Lett. 37, 1214 (2012).

10. P. Rohwetter, J. Kasparian, K. Stelmaszczyk, Z. Hao, S. Henin, N. Lascoux, W. M. Nakaema, Y. Petit, M. Queißer, R. Salamé, E. Salmon, L. Wöste, and J.-P. Wolf, Nat. Photon. 4, 451 (2010).

11. P. Joly, M. Petrarca, A. Vogel, T. Pohl, T. Nagy, Q. Jusforgues, P. Simon, J. Kasparian, K. Weber, and J.-P. Wolf, Appl. Phys. Lett. 102, 091112 (2013).

12. Q. Luo, W. Liu, and S. L. Chin, Appl. Phys. B 76, 337 (2003).

13. W. Chu, H. Li, J. Ni, B. Zeng, J. Yao, H. Zhang, G. Li, C. Jing, H. Xie, H. Xu, K. Yamanouchi, and Y. Cheng, Appl. Phys. Lett. 104, 091106 (2014).

14. Y. Liu, P. Ding, G. Lambert, A. Houard, V. Tikhonchuk, and A. Mysyrowicz, Phys. Rev. Lett. 115, 133203 (2015).

15. H. Xu, E. Lötstedt, A. Iwasaki, and K. Yamanouchi, Nat. Commun. 6, 8347 (2015).

16. C. Li, G.-Q. Liao, and Y.-T. Li, High Power Laser Sci. Eng. 3, e15 (2015).

17. T.-J. Wang, S. Yuan, Y. Chen, and S. L. Chin, Chin. Opt. Lett. 11, 011401 (2013).

18. T.-J. Wang, S. Yuan, Y. Chen, J.-F. Daigle, C. Marceau, F. Théberge, M. Châteauneuf, J. Dubois, and S. L. Chin, Appl. Phys. Lett. 97, 111108 (2010).

19. T. Fujii, A. Zhidkov, M. Miki, K. Sugiyama, N. Goto, S. Eto, Y. Oishi, E. Hotta, and K. Nemoto, Chin. J. Phys. 52, 440 (2014).

20. J. Kasparian, R. Ackermann, Y.-B. André, G. Méchain, G. Méjean, B. Prade, P. Rohwetter, E. Salmon, K. Stelmaszczyk, J. Yu, A. Mysyrowicz, R. Sauerbrey, L. Wöste, and J.-P. Wolf, Opt. Express 16, 5757 (2008).
21. B. Forestier, A. Houard, I. Revel, M. Durand, Y. B. André, B. Prade, A. Jarnac, J. Carbonnel, M. LeNevé, J. C. deMiscault, B. Esmiller, D. Chapuis, and A. Mysyrowicz, AIP Advances 2, 0121511 (2012).

22. X. M. Zhao, J.-C. Diels, C. Y. Wang, and J. M. Elizondo, IEEE J. Quantum Electron. 31, 599 (1995).

23. H. Pépin, D. Comtois, F. Vidal, C. Y. Chien, A. Desparois, T. W. Johnston, J. C. Kieffer, B. La Fontaine, F. Martin, F. A. M. Rizk, C. Potvin, P. Couture, H. P. Mercure, A. BondiouClergerie, P. Lalande, and I. Gallimberti, Phys. Plasmas 8, 2532 (2001).

24. M. Clerici, Y. Hu, P. Lassonde, C. Milián, A. Couairon, D. N. Christodoulides, Z. Chen, L. Razzari, F. Vidal, F. Légaré, D. Faccio, and R. Morandotti, Sci. Adv. 1, e1400111 (2015).

25. L. M. Ball, Appl. Opt. 13, 2292 (1974).

26. J. R. Greig, D. W. Koopman, R. F. Fernsler, R. E. Pechacek, I. M. Vitkovitsky, and A. W. Ali, Phys. Rev. Lett. 41, 174 (1978).

27. J. R. Greig, D. P. Murphy, R. E. Pechacek, M. Raleigh, E. Laikin, and S. Hauver, in Digest of Technical Papers of the 5th IEEE Pulsed Power Conference (1985).

28. T. Uchiyama, M. Hirohashi, H. Miyata, and T. Sakai, Laser Kenkyu 16, 267 (1988).

29. J. Sasaki, S. Kubodera, R. Ozaki, and T. Uchiyama, J. Appl. Phys. 60, 3845 (1986).

30. T. Yamanaka, S. Uchida, Y. Shimada, H. Yasuda, S. Motokoshi, K. Tsubakimoto, Z. Kawasaki, Y. Ishikubo, M. Adachi, and C. Yamanaka, Proc. SPIE 3343, 281 (1998).

31. D. Strickland and G. Mourou, Opt. Commun. 56, 219 (1985).

32. S. L. Chin and K. Miyazaki, Japan. J. Appl. Phys. 38, 2011 (1999).

33. M. Rodriguez, R. Sauerbrey, H. Wille, L. Wöste, T. Fujii, Y. B. André, A. Mysyrowicz, L. Klingbeil, K. Rethmeier, W. Kalkner, J. Kasparian, E. Salmon, J. Yu, and J.-P. Wolf, Opt. Lett. 27, 772 (2002).

34. S. Tzortzakis, B. Prade, M. Franco, A. Mysyrowicz, S. Hüller, and P. Mora, Phys. Rev. E 64, 05740 (2001).

35. K. Jérôme and J.-P. Wolf, in Progress in Ultrafast Intense Laser Science (Springer, Berlin-Heidelberg, 2010), p. 109.

36. A. Schmitt-Sody, A. Lucero, D. French, W. P. Latham, W. White, and W. P. Roach, Opt. Eng. 53, 0515045 (2014).

37. T. J. Wang, Y. Wei, Y. Liu, N. Chen, J. Ju, H. Sun, C. Wang, H. Lu, J. Liu, S. L. Chin, R. Li, and Z. Xu, Sci. Rep. 5, 18681 (2015).

38. H. L. Xu, A. Azarm, J. Bernhardt, Y. Kamali, and S. L. Chin, Chem. Phys. 360, 171 (2009).

39. S. L. Chin, H. L. Xu, Y. Cheng, Z. Z. Xu, and K. Yamanouchi, Chin. Opt. Lett. 11, 013201 (2013). 